$\underline{\text { Supporting information }}$

\title{
Optical Properties of CdSe Superlattices
}

\author{
Natalia Zaitseva, Zu Rong Dai, Francisco R. Leon, and Denise Krol
}

Lawrence Livermore National Laboratory, 7000 East Avenue, Livermore, California, 94551

SI-1. Faceted crystals of bulk Se commonly forming in CdSe. In transmission light they may look similar to the faceted CdSe superlattices.

Se crystals formed in CdSe nanocrystal solutions

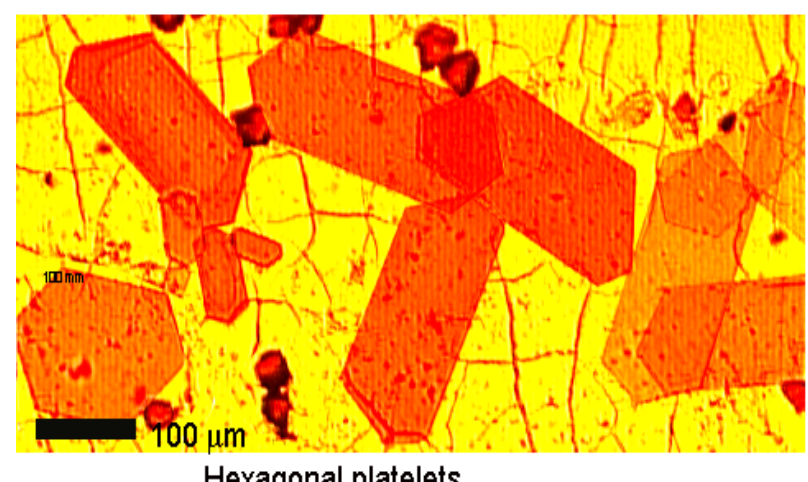

Hexagonal platelets

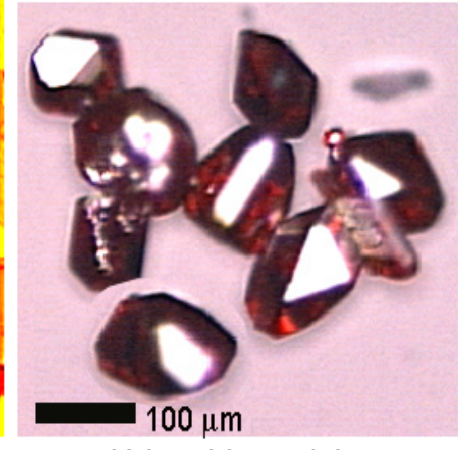

Volumetric crystals

SI-2. Bulk Se crystals are not luminescent, and therefore can be distinguished from superlattices in fluorescence microscope

Se crystals formed in CdSe nanocrystal solutions
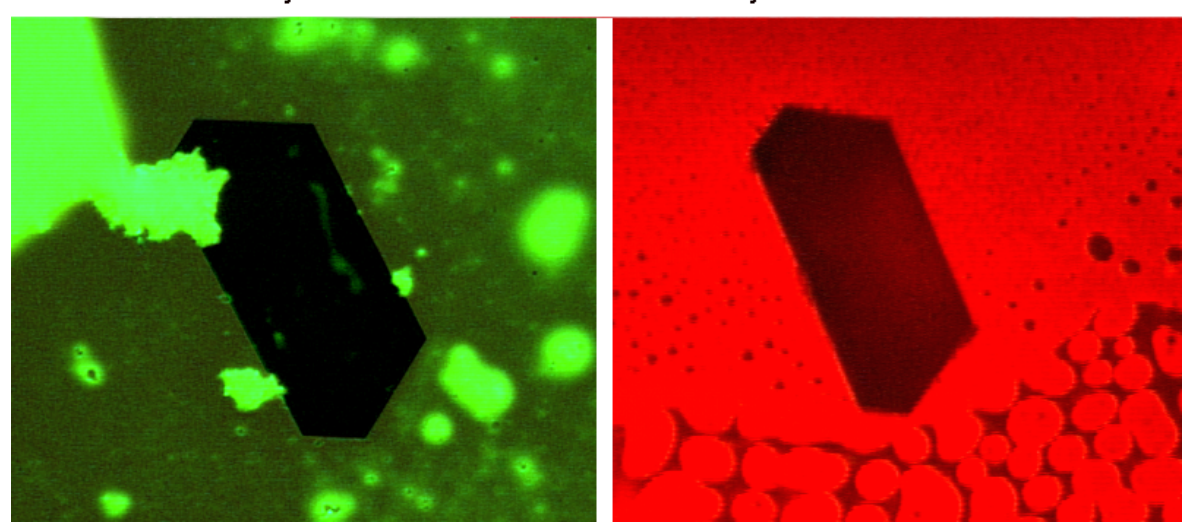
SI-3. Bulk Se crystals belong to monoclinic class of symmetry, and therefore produce bright polarization colors when placed between crossed polarizers

Se crystals formed in CdSe nanocrystal solutions

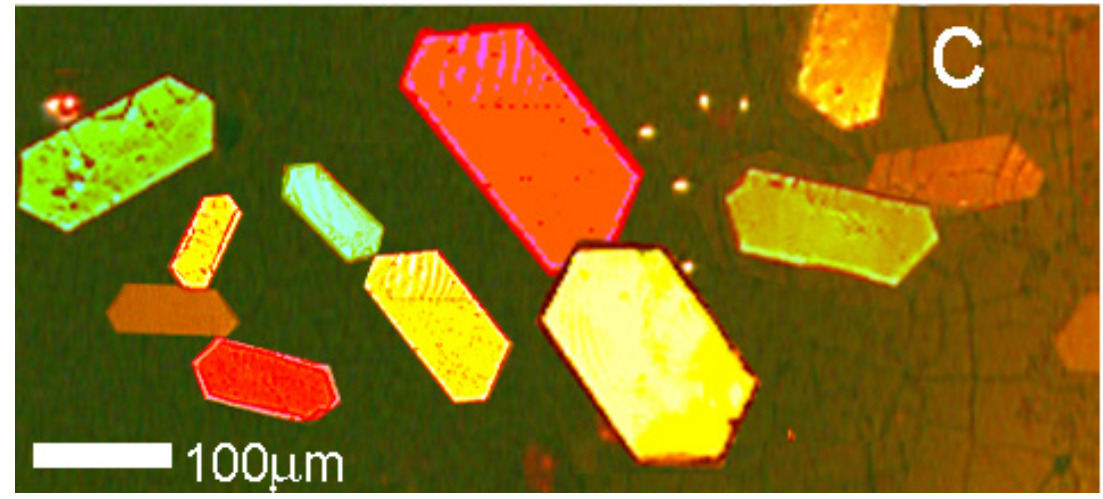

SI-4. CdSe superlattices belong to cubic symmetry, and therefore are dark between crossed polarizers

\section{CdSe superlattices}

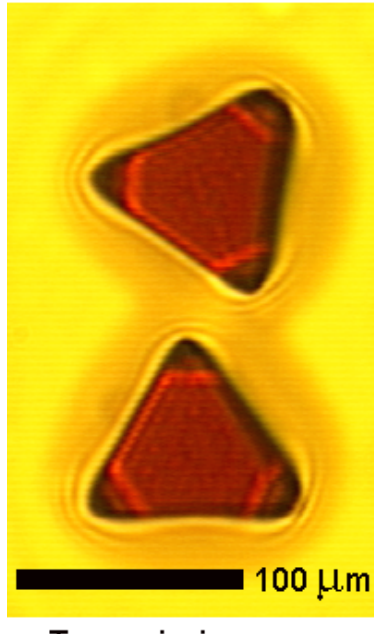

Transmission

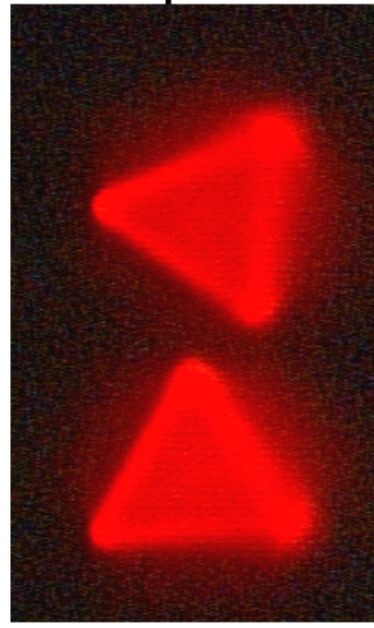

Photoluminescence

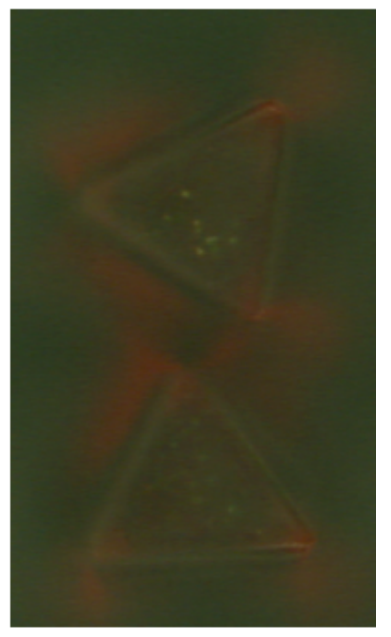

Crossed polarizers 